\title{
TRATAMIENTO DE AGUAS DE ESCORRENTÍA MEDIANTE HUMEDALES ARTIFICIALES: ESTADO DEL ARTE
}

\author{
A RUN-OFF TREATMENT BY MADE WETLANDS - A REVIEW \\ Carlos Andrés Peña Guzmán \\ Ingeniero Ambiental y Sanitario, Candidato a Magister en hidrosistemas \\ Pontifica Universidad Javeriana Sede Bogotá \\ carlos-pena@javeriana.edu.co \\ Jaime Lara Borrero \\ Ingeniero Civil, Ph.D. Profesor Asociado Pontifica Universidad Javeriana \\ laraj@javeriana.edu.co
}

Fecha de recepción: 6 de marzo de 2012

Fecha de aprobación: 20 de noviembre de 2012

\section{RESUMEN}

La concentración de contaminantes en aguas lluvia es un tema que preocupa a las entidades gubernamentales porque deterioran la calidad hídrica de las fuentes receptoras. En varios países, se han iniciado investigaciones y desarrollo de metodologías para tratar este tipo de aguas. Una de estas técnicas es la ejecución de humedales artificiales, y es una de las más aceptadas por su gran eficiencia en la remoción de contaminantes. El presente artículo expondrá el estado del conocimiento, estudiando los métodos de diseño, eficiencias y las nuevas tendencias en la investigación de humedales artificiales que tratan aguas lluvia y/o escorrentía urbana.

Palabras clave: contaminación de aguas lluvias, fuentes difusas, fuentes puntuales, humedales artificiales, mejores prácticas de manejo (BMP), tratamiento de aguas Iluvias.

\begin{abstract}
The pollutant concentration of rainwater is a critical issue for government agencies since impairs the water quality at receiving sources. Some countries have begun research and developed systems for treatment of those waters where made wetland is the most common structure used. This paper offers a review of made wetland to runoff treatment, including design, effectiveness and new trends to research of made wetlands to treat rainwater and/or urban runoff.
\end{abstract}

Keywords: rainwater pollution, diffuse sources, spot sources, made wetlands, best management practices (BMPs), rainwater treatment. 


\section{INTRODUCCIÓN}

Las aguas de lluvia en ambientes urbanizados se recogen para ser vertidas en diferentes cuerpos hídricos, y pueden ayudar como caudales de dilución en cuerpos contaminados y para otros usos humanos; sin embargo, muchas veces no llegan con las condiciones fisicoquímicas óptimas, debido, a que durante el primer intervalo de tiempo en un evento de lluvia, el proceso de escorrentía y el lavado atmosférico aportan contaminantes que se reflejan en las concentraciones de la precipitación, fenómeno conocido como first flush [1-3].

Los contaminantes asociados al first flush en las áreas urbanas, se deben a las diferentes actividades humanas que por el lavado atmosférico y las superficies receptoras (calles, avenidas, parques, cubiertas, etc.), son introducidos al agua por diferentes procesos físicos y químicos [4].

Los usos del suelo generan por consiguiente, diversos contaminantes con magnitudes distintas; así por ejemplo: Ios residenciales, industriales, comerciales y vías de transporte generan sedimentos, nutrientes, materia orgánica y aceites y grasas [5]. Análisis fisicoquímicos y bacteriológicos de escorrentías, realizados por diferentes autores $[1,2,6,7,8]$, y en distintas áreas urbanas, han mostrado la existencia de sólidos suspendidos, coliformes fecales, materia orgánica, metales pesados y otros constituyentes como aceites, grasas e hidrocarburos [8-10].

Como se mencionó, las aguas de lluvia son vertidas a los cuerpos hídricos, y al empeorar sus condiciones naturales, se convierten en puntos de contaminación en las fuentes receptoras [1], ya sea de manera puntual o difusa. La Agencia de Protección Ambiental de los Estados Unidos de América (EPA), estimó que las fuentes no puntuales representaron el 65\% de las cargas totales de contaminación, mientras que para la Administración de Protección Ambiental de Taiwán, contribuyen con más del 20\% de las cargas contaminantes a fuentes hídricas superficiales [1 1].

Debido a la investigación sobre el aporte de contaminantes en las precipitaciones, diferentes entidades y entes educativos hallaron necesario propiciar, el manejo integral de drenajes urbanos y atenuar los impactos ambientales generados, por lo cual se creó el concepto de las mejores prácticas de manejo (BMPs Best Management Practices), también conocidas como SUDS Sustainable Urban Drainage Systems (Estados Unidos), o Sistemas Urbanos de Drenaje Sostenible (Colombia), WSUDS Water Sensitive Urban Design (Australia), LIDS Low Impact Drainage Systems (Estados Unidos), SOIDS [12], Stromwater Quality Improvement Device (Australia), ESD [13], Ecological Sustainable Development (Australia), que consisten en acciones estructurales y no estructurales [14], para el manejo integral del binomio medio ambiente y urbanismo. 
Dentro de las acciones estructurales, los humedales artificiales son uno de los SUDS más usados para la gestión de los contaminantes en escorrentías [15-17], debido a que combinan la eliminación de las concentraciones de diferentes contaminantes, la atenuación de picos [18], y una buena relación costo beneficio [19-22]. Además mejoran la biodiversidad y son poco sensibles a las variaciones de las cargas contaminantes [23-25].

Es importante mencionar que los humedales artificiales para el tratamiento de aguas de Iluvia, tienen diversas tipologías: existen Humedales de Flujo Libre (HFL), Humedales de Flujo Subsuperficial (HFSS), que a su vez se divide en Humedales Subsuperficiales de Flujo Vertical (HSFV), y Humedales Subsuperficiales de Flujo Horizontal (HSFH) [26, 27].

Este sistema de tratamiento natural se ha implementado desde los años 70 en el estado de California, convirtiéndose desde entonces en una práctica muy empleada en Estados Unidos a mediados de los 80 [28].

Hay que señalar que este sistema de tratamiento se ha implementado en varios países, paralelamente al avance de la investigación. Por ejemplo: en Holanda fue diseñado un HSFH con un área de $638 \mathrm{~m}^{2}$ para tratar escorrentías de la ciudad de Ámsterdam; en Australia y Nueva Zelanda se han construido HFL para tratar aguas de lluvia con el propósito de utilizarlas para el riego de campos. En China, se han construido humedales de tipo horizontal para tratar la escorrentía producida en cultivos agrícolas; en Taiwán, se cuenta con plantas piloto de humedales de Flujo Libre y Horizontales que tratan en forma combinada, aguas residuales y aguas de lluvia, en un área de $1.200 \mathrm{~m}^{2}$ construidos en el campus de la Universidad de Sun Yat-Sen [28]. En el aeropuerto de Zurich, existe probablemente uno de los primeros HFSS a escala, con un área $5.500 \mathrm{~m}^{2}$ construido en 1994 para tratar las aguas de escorrentía que se generan al descongelar el hielo de la pistas [29]; igual tipo de tratamiento poseen los aeropuertos internacionales de Pearson en Toronto, Canadá, Edmonton en Alberta, Canadá y en el aeropuerto de Kalmar en Suecia $[30,31]$.

En Bogotá, se han implementado humedales artificiales para proteger los humedales naturales, ya que los colectores pluviales que los alimentan, aportan diferentes concentraciones de contaminantes debido al efecto del primer lavado y la existencia de conexiones sanitarias a estos sistemas, ejecutadas deficientemente. En el humedal de la Conejera, ubicado en la localidad de Suba, se construyó un humedal denominado Hato Chico, que cuenta con un área de drenaje de 4.8 ha, una capacidad de almacenamiento de $1.209 \mathrm{~m}^{3}$ y área superficial de $1.157 \mathrm{~m}^{2}$ (el 2.4\% de la cuenca). Este humedal se diseñó de tipo mixto, compuesto por un HFL y un HFSS. Para la protección del humedal de la 
Conejera, también se montaron dos humedales artificiales de flujo libre llamados Camino Verde Occidental y Oriental; el humedal Occidental presenta un área de drenaje de 0.9 ha, una capacidad de almacenamiento de $65.2 \mathrm{~m}^{3}$ y un área superficial de $86.4 \mathrm{~m}^{2}$ (1\% de la cuencal, mientras que el humedal Oriental cuenta con un área de drenaje de 4.8 ha, una capacidad de almacenamiento de $325.8 \mathrm{~m}^{3}$ y un área superficial de $475.1 \mathrm{~m}^{2}$. Por último, en el humedal Córdoba, se implementó un tratamiento con dos humedales construidos de flujo subsuperficial horizontal en un conjunto residencial Ilamado BoraBora, construidos sobre las torres 1 y 2 del Conjunto. La torre 1 posee tres HFSS que tienen un área de drenaje de 0.1 ha, una capacidad de almacenamiento de $5.5 \mathrm{~m}^{3}$ y un área superficial de $11 \mathrm{~m}^{2}$, mientras que en la torre 3, se creó un HFSS con un área de drenaje de 0.3 ha, capacidad de almacenamiento de $15.4 \mathrm{~m}^{3}$ y un área superficial de $34.2 \mathrm{~m}^{2}$ [32, 33].

Este artículo tiene como objetivo, describir el estado del conocimiento sobre el tratamiento de aguas de escorrentía mediante humedales artificiales, en el cual por consiguiente, se presentarán los criterios de diseño, los rendimientos alcanzados y las nuevas tendencias de investigación.

\section{CRITERIOS DE DISEÑO}

Existe una gran variedad de artículos, libros y textos que describen las condiciones necesarias para un diseño exitoso, con elementos como: el sitio de construcción, análisis hidrológicos, calidad del agua, selección de macrófitas, características del suelo y condiciones geológicas [34]. Por consiguiente, a continuación se describirán algunos criterios que existen en la bibliografía, para diseñar humedales de tratamiento de aguas de lluvia.

Los humedales artificiales se pueden clasificar de acuerdo con varios criterios, pero los más importantes son dos: el régimen de flujo del agua (flujo libre y sub-superficial), y el tipo de macrófita que crece en él [28, 35]. Dentro de esta clasificación, los HFL son los más usados para el tratamiento de la lluvia, debido a su capacidad para controlar cambios en las características del agua [9, 36-38].

Para un buen rendimiento en la remoción de contaminantes, es necesario un pre-tratamiento, ya que diferentes sustancias pueden alterar las condiciones de operación de los humedales y disminuir su rendimiento. Por lo general, los tratamientos primarios utilizados son: trampas para aceites, sedimentadores y canales perimetrales para contención de derrames de aceite [35, 39-41].

Adicionalmente, es importante incluir dentro del diseño, macrófitas robustas, perennes y de fácil propagación [42]; cabe resaltar que esta clase de plantas se encuentra clasificada en dos tipos: (i) platas enraizadas o emergentes y (ii) plantas flotantes [43]. Las macrófitas más utilizadas suelen ser monocultivos de especies emergentes como: Phragmites australis, Eichhornia cras 
sipes, Schoenoplectus validus o Typha spp. Otras especies de macrófitas emergentes se usan en menor medida: Juncus effusus, Sagitaria lancifolia, Scirpus spp., Lemna spp. Por otra parte, las macrófitas sumergidas, como Triglochin spp., se recomiendan por su eficiencia en la remoción de los nutrientes [44].

En el diseño de humedales artificiales que tratan aguas residuales e industriales, las variables: concentración inicial del agua, caudal de entrada y concentración requerida a la salida, son función del diseño y se convierten en datos conocidos. Sin embargo, esta información inicial no es válida en el tratamiento de las aguas de lluvia, debido a las fluctuaciones en los parámetros de entrada (caudal y concentraciones); por lo tanto, algunos métodos de diseño se han presentado de manera empírica [28, 35, 39, 40, 45].

A continuación, se describen algunas metodologías que existen y los factores más relevantes para el diseño y dimensionamiento de humedales para tratar escorrentía.

\subsection{Diseños}

Criterios hidrológicos. Este tipo de énfasis se enfoca en el análisis hidrológico, por representar el factor más importante en el funcionamiento del humedal. Las características hidrológicas son descritas usualmente con la elaboración de un balance hídrico, debido a que proporcionan los vínculos y flujos que existen entre los sistemas hidrológicos, la bioquímica y la ecología del humedal $[34,46]$.

Desde mediados de los 90, investigadores de la Universidad del Estado Norte de Carolina en los Estados Unidos (North Carolina State University NCSU), y colaboradores externos, han instalado más de 30 humedales de aguas de lluvia, y bajo esta experiencia adquirida, elaboraron un grupo de guías para el diseño y construcción de humedales [47].

De acuerdo con estas guías, para la selección apropiada del sitio de construcción del humedal, existen dos factores primordiales; la disponibilidad de agua para alimentar el humedal y la topografía del sitio. Este segundo factor cobra una gran importancia, ya que en lugares planos, la construcción del humedal es mucho más adecuada que en zonas montañosas, por que se reduce el costo por el proceso de excavación.

Es de gran importancia realizar estudios sobre las características físicas del suelo y el nivel freático del lugar, ya que en suelos arenosos se recomienda que el humedal se encuentre $0.15 \mathrm{~m}$ por debajo de la tabla de agua. Para garantizar la existencia de agua en el humedal. En cuanto a suelos arcillosos, se aconseja realizar la base del humedal por encima del nivel freático; sin embargo, en esta base se debe tener una permeabilidad suficientemente baja, con el fin de que el humedal no se seque [20]. Este grupo de investigadores sobre los humedales artificiales, han identificado seis características: 
1. Cámaras de entrega de agua

2. Piscinas profundas: cuya función es retener agua, hasta en temporadas secas.

3. Un sector de aguas poco profundas: donde la vegetación prospera, las plantas pueden bombear oxígeno en su zona radicular y se establecen las condiciones necesarias para la nitrificación. Esta zona representa aproximadamente el 40\% del área del humedal.

4. Un terreno superficial: es un área generalmente seca, que funciona como una zona inundable para la retención de picos.

5. Sector de tierras altas no inundables: estas áreas pueden servir como puntos de observación, si el humedal se utiliza para fines educativos o recreativos.

6. Estructura de salida.

En cuanto al dimensionamiento, para encontrar el tamaño del humedal, se debe tener como eje central el proceso de escorrentía, por que el humedal debe almacenar todo el volumen de agua procedente del primer lavado. En el cálculo de escorrentía se utiliza la siguiente ecuación:

$$
\text { Escorrentía }(\text { pulg })=\frac{(\text { precipitación-0.2S })^{2}}{(\text { precipitación }+0.8)}
$$

Donde,

$$
S=\left(\frac{1000}{\text { Numero de curva }}\right)-10
$$

Numero de curva: Es un indicador de la capacidad de almacenamiento de agua de la cuenca [48]. Luego se calcula el volumen total de escorrentía por tratar en el humedal, empleando la siguiente ecuación:

$$
\text { Volumen a tratar }\left(\frac{\text { acre }}{\text { pulg }}\right)=\frac{\text { área de la cuenca }(\text { acre })}{\text { Escorrentía }(\text { pulg })}
$$

Con el volumen de almacenamiento del humedal conocido, es posible calcular el área superficial necesaria, ya que la profundidad se determina en función de las macrófitas. Las profundidades más utilizadas se encuentran entre 0.15 a 0.3 metros.

También, es importante mencionar que para calcular el tiempo de retención hidráulico sobre el humedal, se debe hacer en función del caudal de salida del mismo y del área de la sección de descarga.

El Centro de Cooperación Investigativa (CRC Cooperative Research Centre), de Australia, es un programa administrado por el Departamento de Industria, Innovación, Ciencia, Investigación y Educación Terciaria, en el cual IOs CRC para la Hidrología de Cuencas y el CRC de Ecología de Agua Dulce, han estado trabajando en el desarrollo de directrices de diseño técnico, para la construcción de humedales y lagunas [49]. 


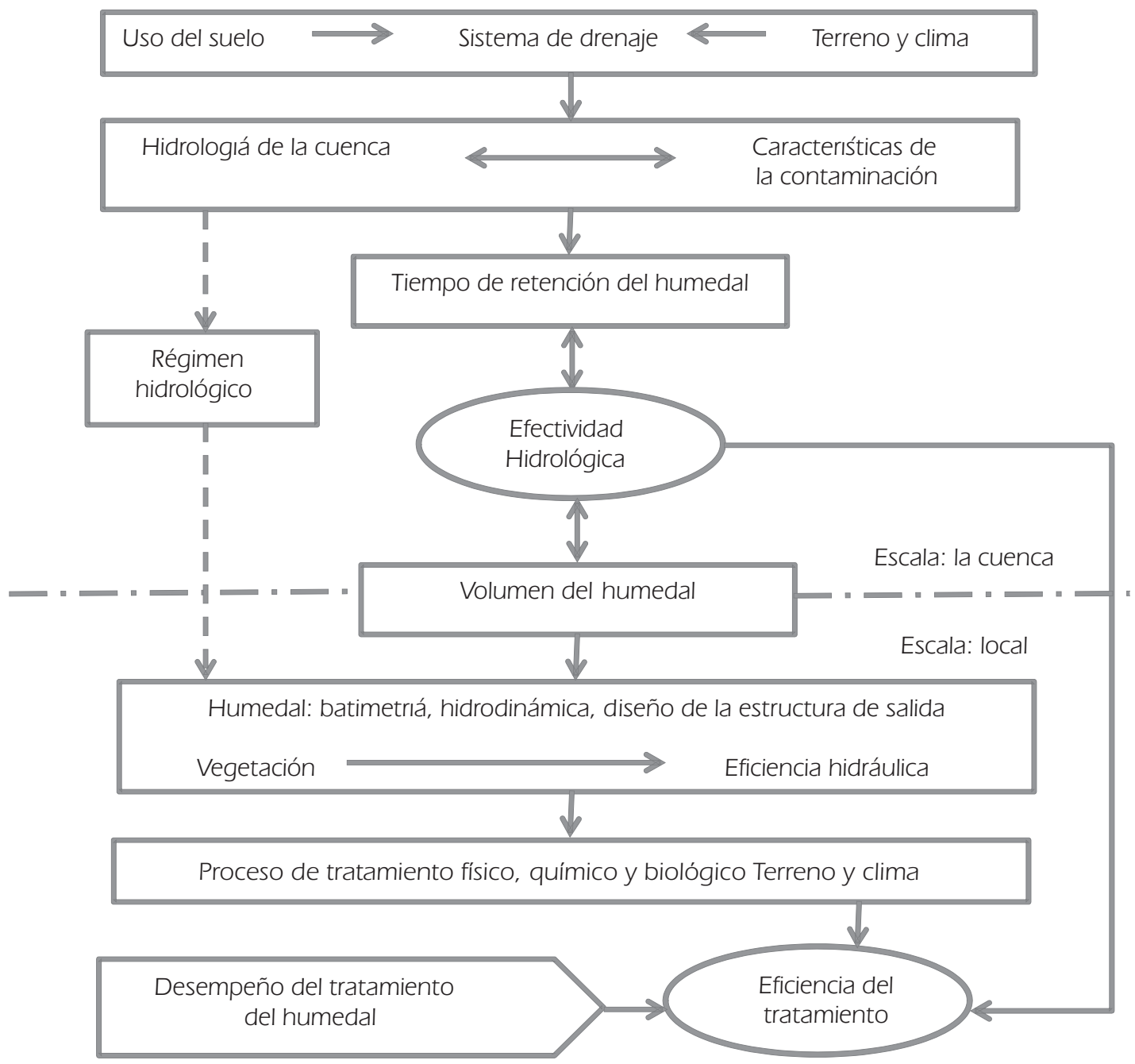

Figura 1. Interacción de elementos para el diseño de un humedal

Fuente: Wong et al., 1998

Para este grupo de investigadores, el diseño exitoso de un humedal artificial de aguas de lluvia, requiere la integración de muchas disciplinas sobre varias escalas espaciales y temporales. Sin embargo, hacen énfasis en tres factores indispensables; efectividad hidrológica, eficiencia hidráulica y régimen hidrológico. En la Figura 1, se ilustra el proceso de diseño e interacción de estos factores, donde la efectividad hidrológica describe la interacción entre la captura de la escorrentía, el tiempo de retención y el volumen del humedal. La eficiencia hidráulica hace referencia a las condiciones en las cuales se mueve el flujo, y por último, el régimen hidrológico describe la variación espacial en periodos largos de la profundidad del agua y en periodos de picos dentro del sistema. 
Shutes et al. $(1999,2004)$ encontraron que el criterio más importante para el dimensionamiento de un humedal artificial, es la selección de la lluvia de diseño, para así determinar el tamaño del humedal y su volumen, ya que este evento será el que transporte el mayor grado de contaminación a tratar. Por lo tanto, y de acuerdo a esta premisa, se puede proyectar para retener eventos cortos o largos de lluvia, con el fin de tratar las cargas contaminantes provenientes del firts flush [35, 39]. A su vez este mismo autor presenta diferentes recomendaciones para los siguientes criterios:

- Tiempo de retención: mínimo de 30 min - máximo de 24 h.

- Relación ancho: largo: 1:4-1:5

- Pendiente del lecho del humedal: $1 \%$ máxima

- Profundidad mínima del substrato $0.6 \mathrm{~m}$

- Conductividad hidráulica del substrato: 10-3 - 10-2 m/s.

- Velocidad del flujo: entre 0.3 a $0.5 \mathrm{~m} / \mathrm{s}$.

De donde una vez ya seleccionada la lluvia de diseño, y elegido el tiempo de retención, se puede calcular el tamaño con la Ley de Darcy, de la siguiente manera $[35,50]$ :

$$
\left(Q_{d} ; \frac{m^{3}}{s}\right)=A_{c} * k_{h} *\left(\frac{\partial H}{\partial x}\right)
$$

Donde $\mathrm{O}_{d}$ es el caudal promedio en $\mathrm{m}^{3} / \mathrm{s}, \mathrm{A}_{\mathrm{c}}$ es el área de la sección transversal del lecho $\left(\mathrm{m}^{2}\right), \mathrm{k}_{\mathrm{h}}$ es la conductividad hidráulica del substrato en $\mathrm{m} / \mathrm{s}$ y $\left(\frac{\partial H}{\partial x}\right)$, es la pendiente o gradiente hidráulico del lecho en $\mathrm{m} / \mathrm{m}$.

Cabe resaltar, que para aplicar la formula 4, es necesario realizar las siguientes dos suposiciones [50]:

- El gradiente hidráulico puede ser usado en lugar de la pendiente.

- La conductividad hidráulica se estabilizará en $1 \times 10^{-3} \mathrm{~m} / \mathrm{s}$ en el humedal.

En la Tabla 1 se presentan otros trabajos relacionados con este criterio de diseño:

Tabla 1. Autores de trabajos relacionados con criterios de diseños

\begin{tabular}{|l|l|}
\hline \multicolumn{1}{|c|}{ AUTOR (AÑO) } & \multicolumn{1}{|c|}{ DOCUMENTO } \\
\hline Koob et al, (1999) & $\begin{array}{l}\text { Hydrologic design considerations of constructed wetlands for urban stormwater } \\
\text { runoff [46]. }\end{array}$ \\
\hline Arnold et al, (2001) & Hydrologic model for design and constructed wetlands [34]. \\
\hline Wong-Somes, (1995) & A stochastic approach to designing wetland for stormwater pollution control \\
\hline Konyha et al, (1995) & Hydrologic design of wetland: advantages of continuous modeling. \\
\hline
\end{tabular}


Criterios hidráulicos. La Water Sensitive Urban Design: Technical Design Guidelines for South East Queensland es una guía creada en Australia, que recomienda llevar a cabo nueve pasos en el diseño de humedales que tratarán escorrentía. Uno de estos pasos es la estimación del caudal de diseño, que recomienda para cuencas menores a 50 hectáreas, aplicar el método racional; por otra parte, si la cuenca es superior a esta área, se debe emplear métodos computacionales para calcular el tránsito de crecientes.

Cabe resaltar que la guía hace énfasis en las condiciones hidráulicas e hidrodinámicas de los humedales, por que considera que estas condiciones son la causa de una excelente eficiencia; por ejemplo: para la relación longitud ancho, recomienda que no sea menor a 5:1, con el fin de obtener unas condiciones hidrodinámicas óptimas.

Adicionalmente, hace un análisis sobre las diferentes formas que puedan tener los humedales, las cuales pueden ser evaluadas en función de un término presentado por Persson et al. (1999) [52], denominado eficiencia hidráulica ( $\lambda$ ), que define las características hidrodinámicas del humedal, de acuerdo con su configuración (Figura 2). Investigadores australianos no recomiendan que se obtengan valores inferiores de 0.5 , sino preferiblemente superiores a 0.7 [51].

$$
\lambda=e\left(1-\frac{1}{N}\right)=\left(\frac{t_{\text {mean }}}{t_{n}}\right)\left(1-\frac{t_{\text {mean }}-t_{p}}{t_{\text {mean }}}\right)=\frac{t_{p}}{t_{n}}
$$

Donde $N$ puede calcularse de dos maneras: la primera de acuerdo con Fogler (1992),

$$
N=\left(\frac{\sigma}{t_{n}}\right)^{-2}
$$

que representa el cuadrado inverso del coeficiente de variación del tiempo de residencia hidráulica de un contaminante, y la segunda de acuerdo con Kadlec y Knight (1996)

$$
N=\frac{t_{n}}{t_{n}-t_{p}}
$$

donde se hace la relación del tiempo al pico y el tiempo de residencia [52]. De acuerdo con lo anterior, $\lambda$ relaciona la forma del humedal, su profundidad y la vegetación del mismo $[53,54]$. 


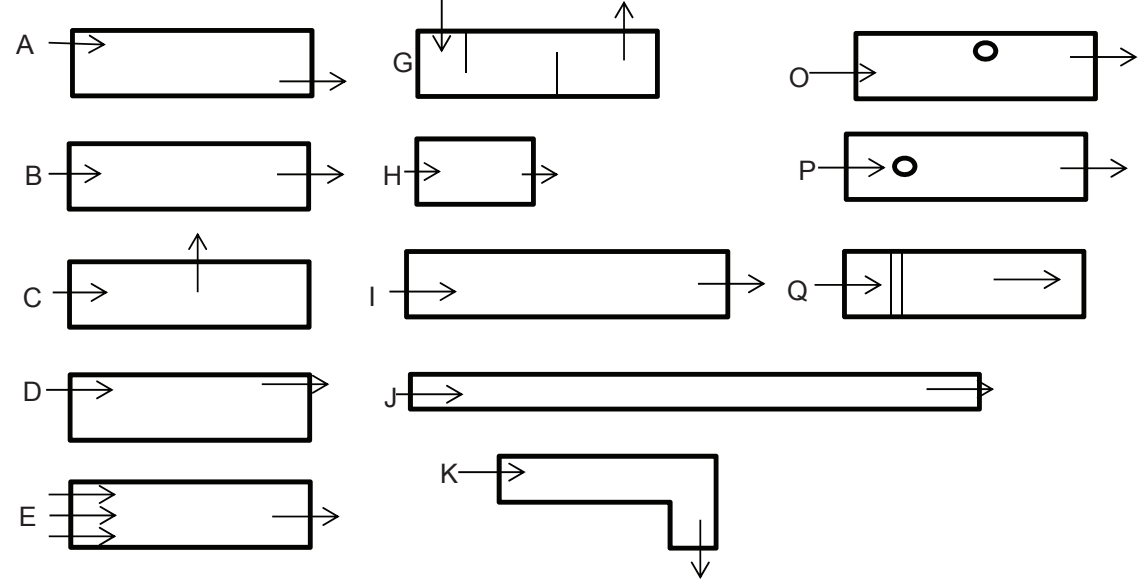

Figura 2. Eficiencia hidráulica de acuerdo con su forma

Fuente: Persson et al., 1998

De los diferentes dimensionamientos evaluados, se encontró:

Tabla 2. Dimensionamientos encontrados por Persson et al. [52]

\begin{tabular}{|l|l|l|}
\hline \multicolumn{1}{|c|}{ Categorías } & \multicolumn{1}{c|}{ Casos } & \multicolumn{1}{c|}{$\lambda$} \\
\hline Pobre eficiencia hidráulica & A, B, C, D, I, H, Ky O & $0.3,0.26,0.11,0.18,0.41,0.11,0.36$ y 0.26 \\
\hline Satisfactoria eficiencia hidráulica & Py O & 0.61 y 0.59 \\
\hline Buena eficiencia hidráulica & E, G y J & $0.76,0.76$ y 0.9 \\
\hline
\end{tabular}

Criterios basados en áreas. El Departamento de Protección Ambiental del Estado de New Jersey, realizó un reporte técnico en 2004, denominado "New Jersey Stormwater Best Management Practices Manual", en donde se presenta un capitulo dedicado al diseño de humedales artificiales.

Para los autores, el parámetro básico para construir un humedal de agua de lluvias, es el volumen de almacenamiento que existe en diferentes zonas del humedal, ya que en general, el volumen total dentro de estas zonas es igual al volumen de escorrentía [55], y por lo tanto, estas zonas son:

Piscina: son zonas que alcanzan profundidades de 0.6 a 1.8 metros, su uso principal es la sedimentación de sólidos, y se dividen en dos clases:

- Estanques: generalmente de profundidades de 1.2 a 1.8 metros, pueden llegar a comprender la mayor parte del humedal.

- Microembalses: poseen la misma profundidad de los estanques, pero su área superficial es menor; por lo general, se encuentran luego de la descarga del sistema pluvial. 
Zonas pantanosas: zonas de menor profundidad con respecto de los estanques, y van de 0.15 a 0.45 metros; por lo general, en esta zona se encuentran las macrófitas emergentes.

Zonas semi-húmedas: es un área casi siempre seca, que funciona como una zona inundable para la retención de picos.

En la Tabla 3, se observan algunos valores recomendados para el dimensionamiento de los diferentes humedales.

Tabla 3. Criterios de diseño para un humedal de aguas de lluvia

\begin{tabular}{|l|l|l|l|}
\hline \multirow{2}{*}{ Características } & \multicolumn{2}{c|}{ Zonas del humedal } \\
\cline { 2 - 4 } & \multicolumn{1}{|c|}{ Estanques } & \multicolumn{1}{c|}{ Pantanosas } & \multicolumn{1}{c|}{ Semi-húmedas } \\
\hline Área de drenaje mínima $\left(\mathrm{m}^{2}\right)$ & 101171.4 & 101171.4 & 40468.5 \\
\hline Relación longitud - ancho mínima & $1: 1$ & $1: 1$ & $1: 1$ \\
\hline
\end{tabular}

Terzakis et al. [52]. Para calcular el tiempo de retención hidráulico para dos diferentes tipos de humedales (HFL y HFSS), utilizaron la ecuación (6):

$$
t=\frac{L W d n}{Q}
$$

Donde $t$ denota el tiempo de retención hidráulico teórico, $L$ la longitud de la cuenca en metros, $W$ el ancho de la cuenca en metros, $d$ la profundidad del agua en metros, $n$ la porosidad de la cuenca y $Q$ el caudal en $\mathrm{m}^{3} / \mathrm{s}$. Para lo cual, el ancho y la longitud fueron calculados mediante la siguiente relación: 1 metro de ancho por 3.5 metros de longitud para el humedal de flujo libre, y para el humedal de flujo subsuperficial se tomó una relación 1:2.

Diferentes propuestas de diseños elaboradas por entidades gubernamentales, universidades o centros de investigación, recomiendan que el dimensionamiento se haga en función del área de la cuenca por drenar. El Ministerio de Medio Ambiente de Ontario recomienda que los humedales sean del orden del 5\% del área de la cuenca; otras investigaciones indican que el humedal podría ser hasta el 1\% del total del área de la cuenca, y se considera aceptable este porcentaje [28]. La Guía de Diseño de Humedales de Maryland de 1987, aconseja que como mínimo, el área del humedal sea igual al 2\% del tamaño de la cuenca vertiente [46].

Por otra parte, Schueler en 1992, presentó algunas técnicas que son implementadas y muy conocidas dentro de los diseños, con el objetivo de mejorar las eficiencias en la remoción de contaminantes: 
- Aumentar el volumen de escorrentía por tratar

- Aumentar la relación de área superficial y volumen

- Incrementar la trayectoria del flujo a través del humedal

- Proporcionar un pre-tratamiento y disipar energía al fluido

- Utilizar vías de eliminación de contaminantes (incrementar tiempos de retención) [56, 57].

Adicionalmente, en la Tabla 4 se presentan algunos criterios de diseños sobre diferentes zonas del humedal. Estos criterios a su vez, son referenciados y utilizados dentro del Manual de Manejo de Aguas de Lluvia de la Universidad Estatal de lowa.

Tabla 4. Criterios de diseño para humedales de aguas de lluvias de acuerdo a Schueler et al. [56]

\begin{tabular}{|c|c|c|c|c|}
\hline ELEMENTO & $\begin{array}{c}\text { SUPERFICIE } \\
\text { PANTANOSA }\end{array}$ & ESTANOUE & $\begin{array}{c}\text { ZONA DE PANTANOS } \\
\text { MENORES }\end{array}$ & $\begin{array}{c}\text { ZONA DE } \\
\text { INUNDACIÓN }\end{array}$ \\
\hline $\begin{array}{c}\text { Relación humedal/ } \\
\text { cuenca }\end{array}$ & 0.2 & 0.01 & 0.01 & $4046.8-40468.5$ \\
\hline $\begin{array}{c}\text { Área mínima de drenaje } \\
\left(\mathrm{m}^{2}\right)\end{array}$ & 101171.4 & 101171.4 & 40468.5 & $1: 1$ \\
\hline $\begin{array}{c}\text { Relación } \\
\text { longitud / ancho }\end{array}$ & $1: 1$ & $1: 1$ & $1: 1$ & \\
\hline
\end{tabular}

Fuente: lowa State University, [57].

Verhoeven [28], reporta diferentes relaciones entre áreas de humedales construidos y áreas de cuenca para diferentes tipos de aguas de escorrentía en diferentes lugares, variando entre el 0.04 y el 15.9\%. Otros autores (Tabla 5) presentan diferentes tipos de áreas y dimensiones.

Tabla 5. Autores que presentan diferentes tipos de áreas y dimensiones

\begin{tabular}{|c|c|}
\hline AUTORES & DOCUMENTO \\
\hline Melbourne Water, (2005) & Constructed wetland systems design guidelines for developers. \\
\hline Mungasavilli et al., 2006 & Constructed Wetlands for Stormwater Management: A Review. \\
\hline Portland (2008) & Stormwater Management Manual \\
\hline
\end{tabular}

Criterios basados en concentraciones. Por otra parte, es importante mencionar que ciertos investigadores han propuesto deducir tasa ad hoc, para humedales con condiciones específicas. Así, un humedal que trataba aguas de escorrentía de las pistas del Aeropuerto de Búfalo, fue diseñado para remover 4.500 kg/día de Demanda Biológica de Oxigeno $\left(\mathrm{DBO}_{5}\right)$, con base en evaluaciones hechas sobre un humedal piloto, del cual se dedujeron tazas de degradación [58], con excelentes resultados. 
Un ejemplo de lo anterior, cuando se quiso diseñar en función de las concentraciones esperadas, fue propuesto por Tilley y Brown [59], que plantearon una metodología, en donde se usaron las concentraciones y el volumen de escorrentía por cada cuenca de drenaje, mediante las ecuaciones (7) y (8):

$$
O_{i j}=0.001 * T C_{i} * R O_{j}
$$

Donde $O_{i j}$ es la masa de salida del contaminante $i$ objetivo en la cuenca $j$ (kg/día); $T C_{i}$ es la concentración objetivo del contaminante $i ; R O_{j}$ es el volumen para la cuenca $j$ ( $\mathrm{m}^{3} /$ día). La masa eliminada se calculó al restar, la masa de entrada de la masa de salida:

$$
R_{i j}=I_{i j}-O_{i j}
$$

Donde $R_{i j}$ es la masa suprimida del contaminante $i$ en la cuenca $j$ (kg/día); $I_{i j}$ es la masa de entrada del contaminante $i$ en la cuenca $j$ (kg/día); se aclara que la cuenca se mide en hectáreas.

Como resultado de lo anterior, se propusieron las ecuaciones (9) y (10) para encontrar el área requerida del humedal artificial por contaminante y volumen de escorrentía, para un grupo de barrio:

$$
\begin{array}{r}
W T A_{i j}=\frac{R_{i j}}{U_{i}} \\
W T A_{j}=\frac{R O_{j} * t}{d_{p}}
\end{array}
$$

Donde, $W T A_{i j}$ es el área del humedal requerida para el contaminante $i$ en la cuenca $j$ en (ha); $U_{i}$ es la tasa de absorción para el contaminante $i$ ( $\mathrm{kg} / \mathrm{ha}$ día). Para el volumen $W T A_{j}$ el área requerida por cuenca $j$ (ha); $R O_{j}$ es el volumen de escorrentía $\left(\mathrm{m}^{3} /\right.$ día); $d_{p}$ es la profundidad y $t$ es el tiempo de residencia (días) [59].

No obstante, es importante mencionar que se han realizado investigaciones que intentan utilizar metodologías aplicadas para el diseño de humedales que tratan aguas residuales, en humedales que tratan aguas Iluvias; por ejemplo: el modelo $k-C^{*}$ que utiliza dos parámetros, la tasa constante k (adimensional), y la concentración de fondo de un contaminante $C^{*}(\mathrm{mg} / \mathrm{L})$ [36, 60, 61, 62].

Por ejemplo: Wing y Geiger (1997), utilizando el modelo $k-C^{*}$ dimensionaron un humedal para el manejo de aguas lluvias, utilizando las ecuaciones (11) y (12):

$$
A=\frac{Q}{k} \ln \left(\frac{c_{i}-c^{*}}{c_{0}-c^{*}}\right)
$$




$$
{ }_{d}=\frac{h}{k} \ln \left(\frac{c_{i}-c^{*}}{c_{0}-c^{*}}\right)
$$

Donde $c_{i}$ es la concentración de entrada, $c_{0}$ es la concentración de salida del humedal, $t_{d}$ tiempo de retención y $h$ es la profundidad del humedal.

\section{EFICIENCIAS Y DESEMPEÑOS EN EL TRATAMIENTO DE LAS AGUAS DE LLUVIA}

Todos los tipos de humedales (naturales y construidos), que se utilizan para el tratamiento de contaminantes, combinan procesos biológicos, químicos y físicos, cuyos mecanismos incluyen la adsorción, la precipitación química, y la absorción [63].

El funcionamiento de los diferentes humedales en el tratamiento de las aguas de lluvia, depende del afluente o carga hidráulica y el tiempo de retención, que a su vez, depende de la intensidad de la lluvia, del volumen de escorrentía y del tamaño del humedal $[64,65]$, todo lo cual puede variar la eficiencia de los procesos anteriormente mencionados dentro del humedal.

Los estudios encontrados durante la revisión de la bibliografía, muestran resultados de remoción de diferentes parámetros contaminantes después del paso por el humedal; así por ejemplo: los parámetros de fósforo y nitrógeno (nutrientes), son de los más investigados, por el alto impacto que generan sobre las masas de agua [66].

En el Sur de la Florida, se han construido varios humedales para el tratamiento específico de nutrientes que se vierten en los Everglades, Abtew et al. [67], encontró que las eficiencias de eliminación del fósforo a una tasa entre 0.48 y $2.72 \mathrm{~g} / \mathrm{m}^{2} / a n ̃ o$, alcanzó el 71\% [67]. Por otra parte, estudios en Finlandia sobre en el humedal Hovi, mostraron rendimientos en la remoción de fósforo entre el 49 y el 68\% [68]. Sin embargo, en Sídney (Australia), se halló una eficiencia muy baja con respecto de lo esperado, al obtenerse un valor del 12\% [63]. Shutes [35], reportó que en Estados Unidos, los humedales HFL cuentan con una remoción muy similar a los humedales de Flujo Sub-Superficiales (HFSS) (50.4\% y 50.2\%).

En cuanto al nitrógeno, se encontraron en Australia eliminaciones del 16\% [63], aunque otros autores indican remociones del 45\% [69]. Shutes [35], expuso que los humedales de HFL presenta una remoción del 63.3\%, superiores a las encontradas en HFSS, en donde se hallaron eficiencias del 44.6\%. Sin embargo, en Kansas se lograron eficiencias muy altas del 85\% [70], y experimentos inducidos muestran que las remociones de amonio son exitosas para HSFV [71, 72]. 
La reducción de sólidos es uno de los parámetros que mayor grado de eliminación tiene, su decaimiento se presenta de forma exponencial con la distancia, debido a los flujos lentos que se dan en los humedales [73]; estas investigaciones han encontrado rendimientos entre el 78 y 90\% [35].

En cuanto a los metales pesados, en los humedales existen cuatro mecanismos de eliminación: (1) adsorción, (2) precipitación, (3) absorción y (4) la sedimentación [74]. Sin embargo, Walker y Hurl [75], encontraron que los metales son removidos principalmente por la sedimentación [75]. Este mismo estudio encontró eficiencias de remoción en cobre $(\mathrm{Cu})$ de $82 \%$, mercurio $(\mathrm{Hg})$ de 86\%, plomo (Pb) de 89\%, y de zinc (Zn) el 47\% [76]. Shutes et al. [35], presentaron eficiencias en el Reino Unido al tratamiento de escorrentía urbana en Pb del 62\%, Zn del 57\% y Cu del 51\%. Por otra parte Scholes et. al. [77], compararon la eficiencia de un humedal en temporada seca y húmeda, y encontraron que en tiempo seco, se alcanzan valores muy bajos en la eliminación de Zn, Pb y Cu; en cuanto a la temporada lluviosa, los porcentajes de remociones fueron muy altos y alcanzaron el 100\% para los mismos parámetros [77].

La supresión de bacterias involucra diferentes procesos como son: la filtración, la radiación solar, la sedimentación, la agregación, la oxidación, la antibiosis, la depredación y la competencia [78]; algunas investigaciones muestran porcentajes de eliminación en estreptococos de 80\%, coliformes 90\% [36] y E. coli de 87\% [79].

Es importante mencionar que la eficiencia de eliminación de este tipo de contaminantes, depende en gran medida de las concentraciones de entrada, la carga hidráulica [74], de los diferentes factores climáticos [80], y de los diferentes tipos de criterios de diseño hechos en cada investigación consultada, situación que muestra las diferencias en los porcentajes de remociones en los datos anteriormente mencionados.

\section{INVESTIGACIONES EN LA ÚLTIMA DÉCADA Y A FUTURO}

En la última década, las investigaciones se han venido centrando en encontrar las eficiencias en la remoción de los diferentes tipos de compuestos tratados por humedales artificiales; por ejemplo: pesticidas, aceites minerales e hidrocarburos policíclicos aromáticos (HPAs), que suelen encontrarse en escorrentía urbana y agrícola.

Es importante mencionar que este tipo de compuestos no se encuentra en todos los casos en los caudales de entrada (especialmente de aceites minerales y HPAs), y que sus concentraciones son muy pequeñas, lo cual hace que sea difícil detectarlos a la salida del humedal [81].

No obstante lo señalado en el párrafo anterior, hay estudios sobre HFL y HFSS, en los cuales se encontró que para HPAs las mayores eficiencias estaban en los HFSS, que alcanzaron hasta el 70\% y en cuanto a HFL, hasta el 50\% [82]. 
En materia de pesticidas, se presenta un campo de investigación más amplio, ya que es común el uso de humedales artificiales para el control de los pesticidas provenientes de la escorrentía de agricultura, ganadería y recreación (campos de golf), y a la vez, está ganando aceptación como un SUDS [83]. En Suráfrica, se hizo una evaluación a un humedal construido para controlar la contaminación no puntual por pesticidas, y se halló que ninguno de los compuestos analizados fueron detectados a la salida, bajo diferentes condiciones climáticas [24], situación parecida en un campo de golf en la India [84], y en diferentes humedales en Francia, donde se alcanzaron remociones del 100\% para diferentes estaciones del año [85].

\section{CONCLUSIONES}

Los humedales artificiales han demostrado ser muy eficientes para el control de contaminantes de aguas de lluvia y de escorrentía, tanto en áreas urbanas como agrícolas, lo cual ha llevado a su gran aceptación como herramienta dentro de los SUDS.

A pesar de que los porcentajes de eliminación de contaminantes encontrados en la literatura son altos, en algunas investigaciones no ha sido así, debido a la variabilidad en los criterios de diseños y la variación en las condiciones climáticas que tienen influencia en los procesos de eliminación de contaminantes.

Por otra parte, es claro que la eficiencia en el tratamiento de pesticidas con humedales artificiales, llega a ser muy alta; en algunos casos del 100\%, mostrando que este tipo de tratamientos amplía su margen de aplicación, no sólo a aguas residuales, industriales o de lluvia, sino también a las que tienen su origen en procesos agrícolas o pecuarios.

\section{REFERENCIAS BIBLIOGRÁFICAS}

[1] Saget A., et al. (1996). The first flush in sewer systems. In: Water Science \& Technology, Vol. 33, pp. 101-109.

[2] Lee J., et al. (2002). First flush analysis of urban storm runoff. In: Science of the Total Environment, The, Vol. 293, pp. 163-175.

[3] Skipworth P.J.T.S.J., and Saul A.J., (2000). The first foul flush in combined sewers: an investigation of the causes. In: Urban Water, Vol. 2, pp. 317-325.

[4] Ashley R.M.B.K.J.L., Hvited-Jacobsen T., and Verbanck M., (2004). Solids in sewers characteristics, effects and control of sewer solids and associated pollutants. In: Scientific and Technical Report Vol. 14. 
[5] Gan J., (2004). Wetland Ecosystems for Treatment of Stormwater in an Urban Environment. In: Wetlands ecosystems in Asia: function and management, Vol. 1, p. 383.

[6] Bertrand J.L., et al., (1998). Distribution of pollutant mass vs volume in stormwater discharges and the first flush phenomenon. In: Water Research, Vol. 32, pp. 2341-2356.

[7] Deletic A., (1998). The first flush load of urban surface runoff. En: Water Research, Vol. 32, pp. $2462-2470$.

[8] Lee J.H. and Bang K.W., (2000). Characterization of urban stormwater runoff. In: Water Research, Vol. 34, pp. 1773-1780.

[9] Scholz M., (2006). Wetland systems to control urban runoff: Elsevier Science.

[10] Scholz M., (2004). Treatment of gully pot effluent containing nickel and copper with constructed wetlands in a cold climate. In: Journal of Chemical Technology \& Biotechnology, Vol. 79, pp. 153-162.

[11] Kao C., et al., (2001). Application of a constructed wetland for non-point source pollution control. In: Water Science and Technology, pp. 585-590.

[12] Begum S., et al., (2008). A comparative review of stormwater treatment and reuse techniques with a new approach: Green Gully. In: WSEAS Transactions on environment and development, Vol. 4, pp. 1002-1013.

[13] Harding R., (2006). Ecologically sustainable development: origins, implementation and challenges. En: Desalination, Vol. 187, pp. 229-239.

[14] Tsihrintzis V.A. and Hamid R., (1997). Modeling and management of urban stormwater runoff quality: a review. In: Water Resources Management, Vol. 11, pp. 137-164.

[15] Carleton J. N., et al., (2000). Performance of a constructed wetlands in treating urban stormwater runoff. In: Water Environment Research, Vol. 72, pp. 295-304.

[16] Braskerud B., et al., (2005). Can constructed wetlands reduce the diffuse phosphorus loads to eutrophic water in cold temperate regions. In: J. Environ. Qual, Vol. 34, pp. 2145-2155.

[17] Bavor H., et al., (2001). Stormwater treatment: do constructed wetlands yield improved pollutant management performance over a detention pond system. In: Water science and technology: a journal of the International Association on Water Pollution Research, Vol. 44, p. 565. 
[18] Bingham D., (1993). Urban runoff pollution prevention and control planning: EPA.

[19] Weiss P.T., et al., (2007). Cost and pollutant removal of storm-water treatment practices. In: Journal of Water Resources Planning and Management, Vol. 133, 218 p.

[20] Hunt W.F. and Doll B.A., (2000). Urban Waterways: Designing Stormwater Wetlands for Small Watersheds. North Carolina Cooperative Extension Service.

[21] Meyer J.L., (1985). A detention basin/artificial wetland treatment system to renovate stormwater runoff from urban, highway, and industrial areas. In: Wetlands, Vol. 5, pp. 135-146.

[22] Kennedy G. and Mayer T., (2002). Natural and constructed wetlands in Canada: An overview. In: Water Quality Research Journal of Canada, Vol. 37, pp. 295-325.

[23] Scholz M. and Lee B., (2005). Constructed wetlands: a review. In: International journal of environmental studies, Vol. 62, pp. 421-447.

[24] Schulz R. and Peall S. K. C., (2001). Effectiveness of a constructed wetland for retention of nonpoint-source pesticide pollution in the Lourens River catchment, South Africa. In: Environmental science \& technology, Vol. 35, pp. 422-426.

[25] Raisin G. and Mitchell D., (1995). The use of wetlands for the control of non-point source pollution. In: Water Science and Technology, Vol. 32, pp. 177-186.

[26] EPA U.S.E.P.A., (1993). Subsurface Flow Constructed Wetlands for Wastewater Treatment .

[27] EPA U.S.E.P.A., (1999). Free Water Surface Wetlands for Wastewater Treatment.

[28] Verhoeven J.T.A., (2006). Wetlands and natural resource management: Springer Verlag.

[29] Vymazal J., (2009). The use constructed wetlands with horizontal sub-surface flow for various types of wastewater. In: Ecological Engineering, Vol. 35, pp. 1-17.

[30] Higginsl J., et al., (2011). The Design \& Operation of a Very Large Vertical Sub-Surface Flow Engineered Wetland to Treat Spent Deicing Fluids and Glycol-Contaminated Stormwater at Buffalo Niagara International Airport. In: Water Practice and Technology, Vol. 6.

[31] Thoren A., et al., (2003). Transport and transformation of de-icing urea from airport runways in a constructed wetland system. In: Wetland Systems for Water Pollution Control VIII, Vol. 48, pp. 283-290. 
[32] Lara B.J., (2010). Humedales construidos para el control de la contaminación proveniente de la escorrentía urbana. En: Acodal, Vol. 226, pp. 19-27.

[33] Lara B.J., (2010). Humedales construidos en la gestión sostenible del sistema pluvial de Bogotá. III Congreso Internacional sobre gestión y tratamineto integral del agua, Argentina.

[34] Arnold J.G., et al., (2001). Hydrologic model for design and constructed wetlands. In: Wetlands, Vol. 21, pp. 167-178.

[35] Shutes R., et al., (2004). Urban and highway runoff treatment by constructed wetlands. In: Wetlands ecosystems in Asia: function and management, Vol. 1, $361 \mathrm{p}$.

[36] Kadlec R.H. and Wallace S., (2009). Treatment wetlands: CRC.

[37] Vymazal J., et al., (2006). Constructed wetlands for wastewater treatment. In: Wetlands and Natural Resource Management, pp. 69-96.

[38] Mungasavalli D.P., and Viraraghavan T., (2006). Constructed wetlands for stormwater management: a review. In: Fresenius Environmental Bulletin, Vol. 15, pp. 1363-1372.

[39] Shutes R., et al., (1999). The design of vegetative constructed wetlands for the treatment of highway runoff. In: The Science of the Total Environment, Vol. 235, pp. 189-197.

[40] Shutes R., et al., (1997). The design of wetland systems for the treatment of urban run off. In: Water Science and Technology, Vol. 35, pp. 19-25.

[41] Shutes R.B.E., et al., (2001). An experimental constructed wetland system for the treatment of highway runoff in the UK. In: Water Science and Technology, pp. 571-578.

[42] Bonilla-Warford C.M. and ZEDLER J.O.Y.B., (2002). Potential for using native plant species in stormwater wetlands. In: Environmental management, Vol. 29, pp. 385-394.

[43] Greenway M. and Davies B., (2003). Stormwater wetlands: design criteria to maximise water quality improvement and minimise mosquito breeding. In: Stormwater Industry Association NSW 7th Regional Conference on Urban Stormwater Managing.

[44] Pérez-Olmedilla M. and Rojo C., (2000). Función depuradora de los humedales I: una revisión bibliográfica sobre el papel de los macrófitos. En: Humedales mediterráneos, Vol. 1, pp. 115-122.

[45] Shutes R.B.E., (2001). Artificial wetlands and water quality improvement. In: Environment international, Vol. 26, pp. 441-447. 
[46] Koob T., et al., (1999). Hydrologic design considerations of constructed wetlands for urban stormwater runoff. In: JAWRA Journal of the American Water Resources Association, Vol. 35, pp. 323-331.

[47] Hunt W., et al. (2010). Stormwater Wetland Construction Guidance. Online: http://mww.bae. ncsu.edu/stormwater/PublicationFiles/WetlandConstruction2010.pdf.

[48] Natural Resources Conservation Service. (1986). Urban hydrology for small watersheds. US Dept. of Agriculture, Soil Conservation Service, Engineering Division, Washington D.C.

[49] Wong T.H.F., et al., (1998). Managing urban stormwater using constructed wetlands: Cooperative Research Centre for Catchment Hydrology.

[50] Magmedov V.G., et al., (1996). The use of constructed wetlands for the treatment of run-off and drainage waters: the UK and Ukraine experience. In: Water Science and Technology, Vol. 33, pp. 315-323.

[51] Waterways M.B., (2006). Water Sensitive Urban Design: Technical Design Guidelines for South East Queensland: Moreton Bay Waterways and Catchment Partnership.

[52] Persson J., et al., (1999). Hydraulics efficiency of constructed wetlands and ponds. In: Water Science \& Technology, Vol. 40, pp. 291-300.

[53] Wong T.H.F., (2006). An overview of water sensitive urban design practices in Australia. In: Water Practice \& Technology, Vol. 1.

[54] Wong T.H.F., (2006). Water sensitive urban design-the journey thus far. In: Australian Journal of Water Resources, Vol. 10, pp. 213-222.

[55] Blick S.A., et al., (2004). New Jersey stormwater best management practices manual. New Jersey Department of Environmental Protection, Division of Watershed Management, Trenton.

[56] Schueler T.R. and Team A.R., (1992). Design of Stormwater Wetland Systems: guidelines for creating diverse and effective stormwater wetlands in the mid-Atlantic Region: Anacostia Resoration Team, Dept. of Environmental Programs, Metropolitan Washington Council of Governments.

[57] Iowa State University, (2008). Iowa Stormwater Management Manual. Iowa State University Institute for Transportation. 
[58] Wallace S. and Liner M., (2011). Design and performance of the wetland treatment system at the Buffalo Niagara International Airport. Specialist Group on Use of Macrophytes in Water Pollution Control, $36 \mathrm{p}$.

[59] Tilley D.R. and Brown M.T., (1998). Wetland networks for stormwater management in subtropical urban watersheds1. In: Ecological Engineering, Vol. 10, pp. 131-158.

[60] Knight R. L., et al., (1999). The use of treatment wetlands for petroleum industry effluents. Environmental science \& technology, Vol. 33, pp. 973-980.

[61] Kadlec R., (1999). The limits of phosphorus removal in wetlands. In: Wetlands Ecology and Management, Vol. 7, pp. 165-175.

[62] Wong T. and Geiger W., (1997). Adaptation of wastewater surface flow wetland formulae for application in constructed stormwater wetlands. In: Ecological Engineering, Vol. 9, pp. 187202.

[63] Birch G. F., et al., (2004). Efficiency of a constructed wetland in removing contaminants from stormwater. In: Wetlands, Vol. 24, pp. 459-466.

[64] Carleton J., et al., (2001). Factors affecting the performance of stormwater treatment wetlands. In: Water Research, Vol. 35, pp. 1552-1562.

[65] Scherger D. and Davis J., (1982). Control of stormwater runoff pollutant loads by a wetland and retention basin. International Symposium on Urban Hydrology, Hydraulics and Sediment Control, Lexington.

[66] Hogan D.M. and Walbridge M.R., (2007). Best Management Practices for Nutrient and Sediment Retention in Urban Stormwater Runoff.

[67] Abtew W., et al., (2004). Stormwater Treatment Areas: Constructed Wetlands for Phosphorus Removal in South Florida Surface Waters.

[68] Liikanen A. P., et al., (2004). Phosphorus removal in a wetland constructed on former arable land. In: Journal of environmental quality, Vol. 33, p. 1124.

[69] Lee C., et al., (2009). Nitrogen removal in constructed wetland systems. In: Engineering in Life Sciences, Vol. 9, pp. 11-22.

[70] Henry C., et al., (2003). Application and performance of constructed wetlands for runoff from small open lots. Ninth International Animal, Agricultural and Food Processing Wastes, North Carolina. 
[71] Scholz M. and Hedmark $\AA$., (2010). Constructed wetlands treating runoff contaminated with nutrients. In: Water, Air, \& Soil Pollution, Vol. 205, pp. 323-332.

[72] WuX., et al., (2008). Constructed Wetlands treating Urban Runoff Contaminated with Nitrogen. In: Bioinformatics and Biomedical Engineering. ICBBE 2008. The 2nd International Conference on Shanghai, pp. 3613-3616.

[73] Li Y., et al., (2007). Modelling wet weather sediment removal by stormwater constructed wetlands: Insights from a laboratory study. In: Journal of Hydrology, Vol. 338, pp. 285-296.

[74] Marchand L., et al., (2010). Metal and metalloid removal in constructed wetlands, with emphasis on the importance of plants and standardized measurements: A review. Environmental Pollution.

[75] Walker D. J. and Hurl S., (2002). The reduction of heavy metals in a stormwater wetland. In: Ecological Engineering, Vol. 18, pp. 407-414.

[76] Nelson E., et al., (2006). Metal removal from water discharges by a constructed treatment wetland. In: Engineering in Life Sciences, Vol. 6, pp. 26-30.

[77] Scholes L., et al., (1998). The treatment of metals in urban runoff by constructed wetlands. In: The Science of the Total Environment, Vol. 214, pp. 211-219.

[78] Davies C. and Bavor H., (2000). The fate of stormwater associated bacteria in constructed wetland and water pollution control pond systems. In: Journal of Applied Microbiology, Vol. 89, pp. 349-360.

[79] Díaz F.J., et al., (2010). Efficacy of constructed wetlands for removal of bacterial contamination from agricultural return flows. In: Agricultural Water Management.

[80] Struck S. D., et al., (2006). Performance of stormwater retention ponds and constructed wetlands in reducing microbial concentrations: US Environmental Protection Agency, National Risk Management Research Laboratory, Office of Research and Development.

[81] Bulc and Sajn Slak A., (2003). Performance of constructed wetland for highway runoff treatment. In: Water Science and Technology, Vol. 48, pp. 315-322.

[82] Terzakis S., et al., (2008). Constructed wetlands treating highway runoff in the central Mediterranean region. In: Chemosphere, Vol. 72, pp. 141-149. 
[83] Gregoire C., et al., (2009). Mitigation of Agricultural Nonpoint-Source Pesticide Pollution in Artificial Wetland Ecosystems-A Review. Climate Change, Intercropping, Pest Control and Beneficial Microorganisms, pp. 293-338.

[84] Kohler E., et al., (2004). Nutrient, metal, and pesticide removal during storm and nonstorm events by a constructed wetland on an urban golf course. In: Ecological Engineering, Vol. 23, pp. 285-298.

[85] Maillard E., et al., (2011). Removal of pesticide mixtures in a stormwater wetland collecting runoff from a vineyard catchment. In: Science of the Total Environment, pp. 1-8. 
